included patients. Marsh classification was applied to interpret the biopsy results. We then verified different multiples of Upper Limits of Normal (ULN) IgA tTG against the D2 biopsy results.

Results The total number of adult patients who had positive serology in the study period was 214. After applying the exclusion criteria, D2 biopsies of 58 patients were studied. The age range was 17 to 89 (mean 53.46, median 57). Male female ratio was 23:35. 47 of these patients had positive D2 biopsies (Marsh 2 or above), 3 patients had biopsies consistent with Marsh 1. Positive Predictive Value (PPV) for positive tTG taking different multiples of ULN was as follows.

\begin{tabular}{lll}
\multicolumn{2}{l}{ Abstract PWE-032 Table 1} \\
\hline PPV & $\begin{array}{l}\text { Marsh 2 and } \\
\text { above }\end{array}$ & $\begin{array}{l}\text { Marsh } 1 \text { and } \\
\text { above }\end{array}$ \\
\hline 1XULN & $0.827(48 / 58)$ & $0.879(51 / 58)$ \\
5 XULN & $0.937(30 / 32)$ & $0.968(31 / 32)$ \\
$10 X U L N$ & $0.90(18 / 20)$ & $0.95(19 / 20)$ \\
$>128$ & $1(12 / 12)$ & $1(12 / 12)$ \\
\hline
\end{tabular}

Conclusions The current Gold standard for diagnosis of Coeliac disease is a positive D2 biopsy. This initial study shows that locally agreed cut off for positive serology can be accepted as diagnostic and the need for OGD and D2 biopsies could be avoided. Potentially this could reduce delays in diagnosis and allow patients to commence a gluten free diet more quickly.

\section{PWE-033 ASSESSING GFD ADHERENCE USING URINE GLUTEN IMMUNOGENIC PEPTIDES IN COELIAC DISEASE: FIRST UK PILOT STUDY}

${ }^{1}$ Anupam Rej*, ${ }^{1}$ Elisabeth Baggus, ${ }^{2}$ Graeme Wild, 'David Sanders. 'Academic Unit Of Gastroenterology, Royal Hallamshire Hospital, Sheffield, UK; ${ }^{2}$ Department of Immunology, Sheffield Teaching Hospitals NHS Foundation Trust, Sheffield

\subsection{6/gutjnl-2019-BSGAbstracts.320}

Introduction Adherence to a gluten free diet (GFD) is essential, as poor adherence can lead to persistent villous atrophy and subsequent complications of coeliac disease. We have previously shown ${ }^{1}$ that non-invasive markers of adherence have a poor sensitivity in comparison to the gold standard of duodenal biopsy. As a result of this, we assessed the novel technique of gluten immunogenic peptides (GIP) in the urine.

Methods Patients with coeliac disease both new diagnosis and those referred to secondary care for further evaluation of dietary adherence/disease remission were assessed from September 2018 to December 2018. Patients were tested for GIP using a rapid immunochromatographic test, following the collection of mid-stream urine samples. All patients were also tested for tissue transglutaminase (IgA-TTG) and endomysial antibodies (IgA-EMA) via blood tests. At least 4 duodenal biopsies were taken from D2 and at least one biopsy from the duodenal bulb, with the presence/absence of villous atrophy used to determine sensitivities of the tests.

Results 17 patients were recruited ( $\mathrm{n}=12$ female, 71\%), median age 52 years (range 25-74 years), median duration of GFD 96 months (0-840 months). 2 patients were newly diagnosed CD, 15 patients were established diagnosis of CD. Table 1 outlines the sensitivity and specificity of GIP, IgA-TTG and IgA-EMA.

Conclusions Urine GIP testing was not superior to IgA-TTG or IgA-EMA. Further data is required to assess this modality as a predictor of villous atrophy and adherence before this can be used in clinical practice.

\begin{tabular}{cllll}
\multicolumn{4}{c}{ Abstract PWE-033 Table 1} & \multicolumn{3}{c}{ | Comparison of GIP, TTG and EMA } \\
\hline & $\begin{array}{l}\text { Sensitivity\% } \\
\text { (CI) }\end{array}$ & $\begin{array}{l}\text { Specificity\% } \\
\text { (CI) }\end{array}$ & $\begin{array}{l}\text { Positive } \\
\text { predictive } \\
\text { value\% (CI) }\end{array}$ & $\begin{array}{l}\text { Negative } \\
\text { predictive } \\
\text { value\% (CI) }\end{array}$ \\
\hline \multirow{2}{*}{ GIP } & 66.7 & 63.6 & 50.0 & 77.7 \\
& $(24.1-94.0)$ & $(24.1-94.0)$ & $(17.4-82.5)$ & $(40.1-96.1)$ \\
IgA-TTG & 40.0 & 100.0 & 100.0 & 78.6 \\
& $(7.3-83.0)$ & $(67.9-100.0)$ & $(19.8-100.0)$ & $(48.8-94.3)$ \\
IgA-EMA & 40.0 & 100.0 & 100.0 & 78.6 \\
& $(7.3-83.0)$ & $(67.9-100.0)$ & $(19.8-100.0)$ & $(48.8-94.3)$ \\
& & & & \\
\hline
\end{tabular}

\section{REFERENCE}

1. Lau M, Mooney P, White W, et al. The Role of A Point of Care Test, Simtomax, in Predicting Histological Remission in Coeliac Disease on A Gluten Free Diet. Gut 2016;65:A166-A167.

\section{PWE-034 SHOULD WE BE DIAGNOSING COELIAC DISEASE IN THE ELDERLY?}

${ }^{1}$ Lauren Marks, ${ }^{1}$ Anupam Rej*, ${ }^{1}$ Matthew Kurien, ${ }^{1}$ Michael Rees, ${ }^{2}$ Simon Cross, ${ }^{3}$ Marios Hadjivassiliou, ${ }^{1}$ David Sanders. ${ }^{1}$ Academic Unit of Gastroenterology, Royal Hallamshire Hospital, Sheffield, UK; ${ }^{2}$ Department of Histopathology, Royal Hallamshire Hospital, Sheffield, UK; ${ }^{3}$ Department of Neurology, Royal Hallamshire Hospital, Sheffield, UK

\subsection{6/gutjnl-2019-BSGAbstracts.321}

Introduction Coeliac disease (CD) is common, but is underdiagnosed in the elderly due to lack of physician awareness and heterogeneity of presentation. We aimed to establish whether there has been a change in the diagnosis of CD in the elderly (over 65 years old) from 1990 until present day, as well as the clinical and histopathological features of CD in old vs. young adults.

Methods Newly diagnosed CD patients were prospectively recruited from the Coeliac Specialist Clinic at the Royal Hallamshire Hospital, Sheffield, between 2008 and 2017. All patients had villous atrophy (VA) on biopsy, positive coeliac serology (IgA-tissue transglutaminase and IgA-endomysial antibodies) and compatible Human Leukocyte Antigen (HLA) typing. Additionally, patients were retrospectively identified from 1990 to 2008 to determine the trend in elderly CD diagnostic frequency over time.

Results 1605 patients with CD were recruited ( $n=644$ prospectively, $\mathrm{n}=961$ retrospectively). Of these, 208 patients (13.0\%) were diagnosed over the age of 65 years between 1990 and 2017. The proportion of elderly CD diagnoses increased from $0 \%(n=0 / 11)$ in $1990-1991$ to $18.7 \%(n=41 /$ $232)$ in $2016-2017(\mathrm{p}<0.001)$. The male to female ratio decreased with increasing diagnostic age from 1.71:1 in the 18-34 age group to $1.02: 1$ in the over 65 age group $(\mathrm{p}<0.001)$. Younger patients more commonly presented with fatigue $(\mathrm{p}<0.001)$ and gastrointestinal symptoms including diarrhoea $(p=0.005)$, abdominal pain $(p=0.019)$, and IBStype symptoms $(p=0.008)$, as seen in table 1 . Older people 
more frequently presented with B12 deficiency $(p=0.037)$ and had milder degrees of VA than younger patients $(\mathrm{p}=0.005)$.

Conclusions Coeliac disease is common in elderly patients but gastrointestinal symptoms occur less frequently than in younger individuals. Elderly patients tend to present with a milder degree of VA. This questions the utility of active case finding in this age group, as a gluten free diet may not be the most appropriate management in this cohort.

\begin{tabular}{|c|c|c|c|c|c|}
\hline & \multirow{3}{*}{$\begin{array}{l}\text { Prevalence in } \\
\text { overall prospective } \\
\text { cohort ( } n=644)\end{array}$} & \multicolumn{3}{|c|}{ Age (years) } & \multirow[t]{3}{*}{$p$-value } \\
\hline & & $18-34$ & $35-64$ & $>65$ & \\
\hline & & $(n=259)$ & $(n=287)$ & $(n=99)$ & \\
\hline Fatigue & $24.9 \%(160)$ & $31.9 \%(82)$ & $23.0 \%(66)$ & $12.1 \%(12)$ & $<0.001$ \\
\hline Diarrhoea & $30.4 \%$ (196) & $35.7 \%(92)$ & $30.0 \%(86)$ & $18.2 \%(18)$ & 0.005 \\
\hline Abdominal pain & $23.2 \%(149)$ & $29.2 \%(75)$ & $20.2 \%(58)$ & $16.2 \%(16)$ & 0.019 \\
\hline $\begin{array}{l}\text { IBS-type } \\
\text { symptoms }\end{array}$ & $18.0 \%(528)$ & $24.4 \%(63)$ & $15.0 \%(43)$ & $10.1 \%(10)$ & 0.008 \\
\hline B12 deficiency & $12.1 \%$ (78) & $10.5 \%(27)$ & $10.8 \%(31)$ & $20.2 \%(20)$ & 0.037 \\
\hline
\end{tabular}

\section{PWE-035 GLOBAL TRANSLATION OF COELIAC DISEASE HISTOLOGY AND OTHER GLUTEN RELATED MICROENTEROPATHY}

Kamran Rostami, Mohammad Derakhshan*, Arzu Ensari, Amitabh Srivastava, Vincenzo Villanacci, Michael Marsh, Antonio Carroccio, Umberto Volta, Alessio Fasano, Julio Cesar Bai, Mihai Danciu, David Sanders, Anna Sapone, Carolina Ciacci, Luca Elli, Stefano Guandalini, Marjorie Walker, Laura De Magistris, Hilary Jericho, Sauid Ishaq, Gabriel Becheanu, Carlo Catassi, Sherly Mathews, James Going, Mohammad RostamiNejad, Chris Mulder, Hamid Mohaghegh, Matt Johnson, Geoffrey Holmes, Gabrio Bassotti, Anna Bozzola, Chiara Ricci, Ada Maria Florena, Rachele Delsordo, Roxana Maxim, Prasenjit Das, Govind Makharia, Knut Lundin, Katri Kaukinen, Adam Levene, Nicola Fusco, Afshin Moradi, Giovanni Casella, David Hayman, Camillo Dibella, Catherine Hagen, Giuseppe Mazzarella, Melanie Johncilla, Mehul Lamba, Juha Taavela, Mohammad Reza Zali, Sarah Liprot, Christine Rodger. Department of Gastroenterology, Palmerston North, New Zealand

10.1136/gutjnl-2019-BSGAbstracts.322

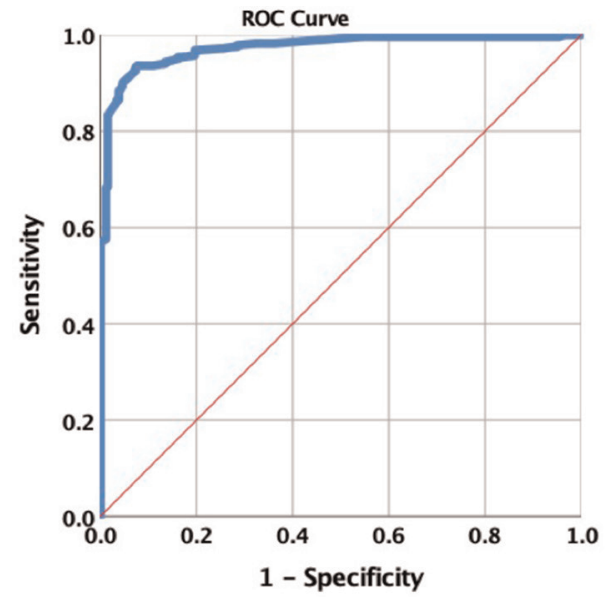

IEL Density for Coeliac
Introduction Intestinal epithelial cell damages generated by inflammation in coeliac disease (CD) ranges from sub-microscopic to severe architectural distortion. Translation of quantitative morphological changes in intestinal microorgans, like villus/crypt transformation, distribution of inflammatory cells and diagnostic cut offs, is lacking for $\mathrm{CD}$ and gluten related micro-enteropathies.

Method Investigators from 22 centres, 9 countries of 4 continents, recruited CD patients with Marsh 0-II histology $(n=299)$, NCGS $(n=151)$, and 262 controls. Based on an agreed protocol, epithelial morphology including intraepithelial lymphocyte (IEL) density, villus height and crypt depth were measured in well-oriented duodenal biopsies.

Results In total 712 subjects were recruited from Australia (20), Finland (20), India (25), Iran (37), Italy (246), Romania (10), Turkey (30), UK (166) and USA (158). Preliminary analyses showed raw IEL density (IEL/100EC) was poorly correlated with tTG, villus height, crypt depth or their ratios, and even significant findings did not show strong correlation coefficients $(<0.36)$. The IEL density cut off scored 93\% sensitivity and specificity at 24/100EC for CD. However, for NCGS the optimal sensitivity and specificity cut off was at 22IEL/ 100EC giving a sensitivity of $57 \%$ and specificity of $80 \%$ (see fig 1). The villus height was significantly shorter in CD compared to either control $(\mathrm{p}<0.001)$ or NCGS groups $(\mathrm{p}<0.001)$. Also, NCGS had short villus height than control $(\mathrm{p}<0.001)$.

Conclusion The most specific and strongest biomarker for CD with microenteropathy is serology acting as the gold standard in this group. Villus height and crypt depth would serve as complementary tools in diagnosis of mild CD and NCGS patients. NCGS seem to have a milder morphological change compared to $\mathrm{CD}$ even when they present with similar Marsh scores. This study also confirms the cut off of IEL for CD with microenteropathy is similar to $\mathrm{CD}$ with severe enteropathy at 25 IEL/100EC.

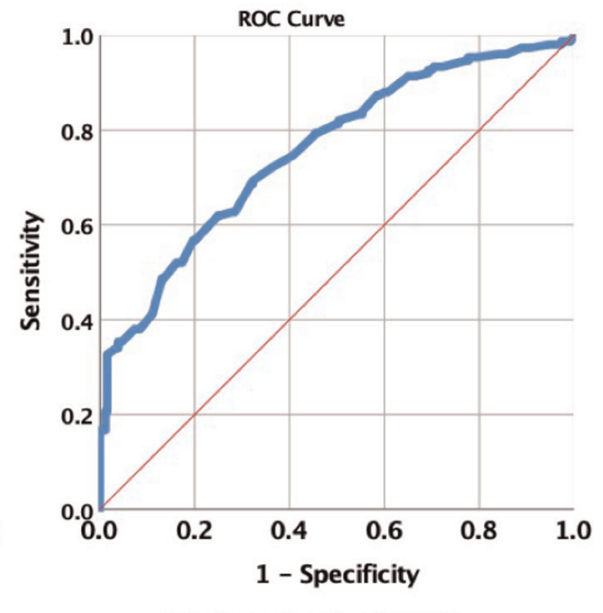

IEL Density for NCGS 